\title{
Diagnóstico molecular da malária em uma unidade de atenção terciária na Amazônia Brasileira
}

\author{
Molecular diagnosing of malaria in a tertiary \\ care center in the Brazilian Amazon region
}

\author{
Mônica Regina Farias Costa ${ }^{1,2}$, Pedro Paulo Ribeiro Vieira ${ }^{1,2}$, Cynthia de Oliveira Ferreira ${ }^{3}$, \\ Marcus Vinícius Guimarães de Lacerda ${ }^{1,2,4}$, Wilson Duarte Alecrim ${ }^{1,4}$ \\ e Maria das Graças Costa Alecrim ${ }^{1,2,4}$
}

\begin{abstract}
RESUMO
0 exame de rotina para o diagnóstico da malária continua sendo a gota espessa, apesar da comprovada diminuição da sensibilidade e especificidade em situações de densidade parasitária baixa e infecções mistas. A reação em cadeia da polimerase vem sendo cada vez mais utilizada para a detecção molecular e identificação das espécies de plasmódio, por apresentar maior sensibilidade e especificidade. Foi realizada a nested-PCR em amostras de sangue total de 344 pacientes com síndrome febril aguda que se apresentaram para o diagnóstico de malária, em uma unidade terciária de saúde, em Manaus (Amazonas). Nenhum caso de malária por Plasmodium malariae foi diagnosticado à gota espessa ou PCR. Observou-se co-positividade de 96,7\%, co-negatividade de 62,2\% e coeficiente kappa de 0,44 entre PCR e gota espessa para Plasmodium falciparum. Para Plasmodium vivax, c0positividade de 100\%, co-negatividade de 78,1\% e coeficiente kappa de 0,56. Na detecção da malária mista, co-positividade de 100\%, co-negatividade de 84,9\% e coeficiente kappa de 0,26. A reação em cadeia da polimerase detectou alto número de infecções mistas nas amostras analisadas, mas seu uso rotineiro no diagnóstico da malária merece ainda ampla discussão.
\end{abstract}

Palavras-chaves: Malária. Diagnóstico. Biologia molecular. Reação em cadeia da polimerase.

\begin{abstract}
The routine test for diagnosing malaria is still the thick blood smear, despite its known decreased sensitivity and specificity in situations of low parasite density and mixed infections. The polymerase chain reaction is increasingly being used for molecular detection and identification of Plasmodium species, due to its higher sensitivity and specificity. Nested PCR was performed on whole-blood samples from 344 patients with acute febrile syndrome who came to a tertiary healthcare center in Manaus (State of Amazonas) for diagnostic confirmation of malaria. No malaria cases caused by Plasmodium malariae were detected through the blood smear or PCR. Co-positivity of $96.7 \%$, co-negativity of $62.2 \%$ and kappa coefficient of 0.44 were observed between PCR and thick blood smear for Plasmodium falciparum. For Plasmodium vivax, co-positivity of 100\%, co-negativity of $78.1 \%$ and kappa coefficient of 0.56 were observed. For mixed infection, co-positivity of $100 \%$, co-negativity of $84.9 \%$ and kappa coefficient of 0.26 were observed. Polymerase chain reaction detected a high number of mixed infections in the samples analyzed, but its routine use for diagnosing malaria still deserves further discussion..
\end{abstract}

Key-words: Malaria. Diagnosis. Molecular biology. Polymerase chain reaction.

0 diagnóstico laboratorial rotineiro da malária é realizado pelo método da gota espessa, consistindo na identificação dos parasitos no sangue periférico, por meio de microscopia óptica. 0 aperfeiçoamento da microscopia e a introdução de corantes biológicos que permitem identificar a espécie, estádio de desenvolvimento, viabilidade e quantificação dos parasitos, tornaram o método simples, rápido e satisfatório quanto à sua sensibilidae e especificidade ${ }^{16}$. Entretanto, discute-se a baixa sensibilidade da técnica para o diagnóstico de baixas parasitemias (comum em portadores assintomáticos de

\footnotetext{
1. Gerência de Malária. Fundação de Medicina Tropical do Amazonas, Manaus, AM. 2. Universidade do Estado do Amazonas, Manaus, AM. 3. Universidade Federal do Amazonas, Manaus, AM. 4. Centro Universitário Nilton Lins, Manaus, AM.

Órgãos financiadores: Superintendência da Zona Franca de Manaus (convênio nº 035/2002) e Conselho Nacional de Desenvolvimento Científico e Tecnológico (convênio nº 550260/2001-3).

Endereço para correspondência: Dr. Pedro Paulo Ribeiro Vieira. Gerência de Malária/FMT-AM. Av. Pedro Teixeira 25, D. Pedro I, 69040-000 Manaus, AM.

Tel.: 5592 2127-3443; Fax: 5592 3238-3762

e-mail: pvieira@fmt.am.gov.br

Recebido para publicação em 14/05/2007

Aceito em 02/07/2008
} 
plasmódio) e de infecções mistas ${ }^{13}$. Desde 1990, vários estudos têm ressaltado a aplicação da reação em cadeia da polimerase (polymerase-chain reaction - PCR) no diagnóstico molecular da malária $^{126}$, nos inquéritos epidemiológicos ${ }^{28} 30$, no rastreamento de doadores infectados em bancos de sangue ${ }^{27}$, na determinação do portador assintomático de plasmódio ${ }^{129} \mathrm{e}$ no monitoramento da resposta terapêutica ${ }^{5}$. Mas apesar das inúmeras experiências demonstrarem que a PCR apresenta maior sensibilidade que o exame microscópico 241824253132 , sendo até utilizado com padrão-ouro no diagnóstico da infecção, por alguns autore ${ }^{20}$, o real desempenho da técnica em campo carece de mais evidências, em especial pelo alto custo e infra-estrutura sofisticada ainda necessários para sua execução. Pela escassez de dados referentes ao desempenho da PCR em áreas endêmicas para malária, no Brasil, este trabalho teve a finalidade de analisar a experiência com o diagnóstico molecular da malária de uma unidade de atenção terciária para doenças infecciosas, em área hiper-endêmica para malária, na Amazônia Brasileira, comparando uma das mais reconhecidas técnicas de detecção de DNA genômico plasmodial com o tradicional diagnóstico pela gota espessa recomendado pela Organização Mundial da Saúde, tendo como objetivos secundários a estimativa da frequiência de infecções mistas e da infecção por Plasmodium malariae.

\section{PACIENTES E MÉTODOS}

Trata-se de um estudo de avaliação da co-positividade e da conegatividade da PCR com a gota espessa, na Fundação de Medicina Tropical do Amazonas (FMT-AM), em Manaus (Amazonas). 0 estudo foi aprovado pelo Comitê de Ética em Pesquisa da FMTAM (processo no 2816/2002) e contou com o consentimento por escrito dos participantes.

Seleção dos pacientes. Foram selecionados aleatoriamente 344 pacientes com síndrome febril aguda, que procuraram a FMT-AM para o diagnóstico de malária, no período de janeiro de 2001 a dezembro de 2002.

Gota espessa. Na FMT-AM, o diagnóstico de rotina da malária é realizado pelo exame da gota espessa (método de Walker), seguindo a padronização da Organização Mundial de Saúde e do Ministério da Saúde do Brasil ${ }^{16}$. 0 diagnóstico da espécie e a quantificação da parasitemia foram realizados por dois técnicos com reconhecida experiência no diagnóstico microscópico de malária, através da contagem do número de parasitas, em campos de grande aumento, por 100 leucócitos. A seguir, a densidade parasitária era convertida para $\mathrm{mm}^{3}$ de sangue, utilizando a leucometria de cada paciente, obtida a partir de hemograma automatizado.

Extração do DNA. 0 DNA genômico plasmodial foi extraído de amostras de sangue total, coletadas destes pacientes. Para a extração do DNA parasitário, foi utilizado o Kit QIAamp DNA Blood Mini Kit ${ }^{\circledR}\left(\right.$ QIAGEN ${ }^{\circledast}$ Cat. $n^{\circ}$ 51106), seguindo as especificações do fabricante e o método que emprega o Chelex ${ }^{\circledast}$. 0 DNA genômico plasmodial foi armazenado em $-20^{\circ} \mathrm{C}$ até a realização da PCR.

Amplificação do DNA. A PCR foi realizada conforme técnica utilizada na FMT-AM, desde 1999, com amplificação de uma região específica do DNA, utilizando-se iniciadores (primers) pré-desenhados e descritos por Snounou e cols ${ }^{25}$, objetivando a amplificação de regiões do gene codificante para a subunidade menor do RNA ribossomal do Plasmodium (ssurRNA). 0 método consiste em uma PCR aninhada (nested-PCR), com primers gênero-específicos na reação primária, e espécie-específicos na reação secundária (Tabela 1). As amplificações foram realizadas em termociclador Eppendorf ${ }^{\circledast}$-Master Cycler Gradient, com total de 30 ciclos, com as seguintes temperaturas: desnaturação: $95^{\circ} \mathrm{C} / 5^{\prime}$; anelamento: $55^{\circ} \mathrm{C} / 2^{\prime}$; e extensão: $72^{\circ} \mathrm{C} / 2^{\prime}$.

Tabela 1 - Seqüência de oligonucleotídeos iniciadores utilizados para o diagnóstico molecular da malária.

\begin{tabular}{lc}
\hline Seqüência de oligonucleotídeos iniciadores & Segmento ssurRNA $(\mathrm{pb})$ \\
\hline Plu5 - 5'CCTGTTGGTGCCTTAAACTTC 3' & 1.200 \\
Plu6 - 5' 'TTAAAATTGTTGCAGTTAAAA 3' & \\
Fal1 - 5'TTAAACTGGTTTTGGGAAAACCAAATATATT 3' & 205 \\
Fal2 - 5'ACACAATGAACTCAATCATGACTACCCGTC 3' & \\
Viv1 - 5'CGCTTCTAGCTTAATCCACATAACTGATAC 3' & 120 \\
Viv2 - 5'ACTTCCAAGCCGAAGCAAAGAAAGTCCTTA 3' & \\
Mal1 - 5'ATAACATAGTTGTACGTTAAGAATAACCGC 3' & 144 \\
Mal2 - 5'AAAATTCCCATGCATAAAAAATTATACAAA 3' & \\
\hline
\end{tabular}

Plu: Plasmodium sp, Fal: Plasmodium falciparum, Viv: Plasmodium vivax, Mal: Plasmodium malariae, pb: pares de bases

Análise de amplicons. Após a amplificação, o produto da PCR foi aplicado em gel de agarose $2 \%$, corado com brometo de etídeo $(10 \mu \mathrm{g} / \mu \mathrm{L})$, e submetido à eletroforese com tampão TEB 1x. A observação dos segmentos de DNA amplificados foi feita em transiluminador, sob luz ultravioleta, e registrado em sistema digital para documentação de gel.

Análise dos resultados. Foram calculadas a co-positividade (equivalente à sensibilidade) e a co-negatividade (equivalente à especificidade) entre os testes, além da avaliação da concordância, pelo coeficiente kappa. A diferença entre médias de densidade parasitária foi estimada pelo teste não-paramétrico de Mann-Whitney.

\section{RESULTADOS}

A positividade global para infecção malárica à gota espessa foi $35,7 \%$. Pela PCR, esta positividade foi $68,3 \%$. Os resultados de gota espessa e PCR estão discriminados na Tabela 2. A ocorrência de infecção mista (Plasmodium falciparum + Plasmodium vivax) foi 1,1\% (4/344) pela gota espessa e 14,8\% (51/344) pela PCR. Na Figura 1A, observa-se um campo de grande aumento (1.000x) de gota espessa de paciente com malária mista, evidenciada pela presença de gametócitos de Plasmodium falciparum e trofozoítos amebóides (formas irregulares) de Plasmodium vivax. Em outra amostra, cuja gota espessa foi positiva apenas para Plasmodium vivax, o gel de eletroforese da PCR (Figura 1B) confirmou o diagnóstico de infecção mista (colunas $4 \mathrm{e}$ 5). A PCR, portanto, detectou 13,4 vezes mais casos de infecção mista, em comparação com a gota espessa. Nenhum caso de malária por Plasmodium malariae foi diagnosticado pela gota espessa ou pela PCR. Comparando gota espessa e PCR na detecção de Plasmodium falciparum, observou-se 
Tabela 2 - Descrição do diagnóstico microscópico pela gota espessa e diagnóstico molecular pela nested-PCR.

\begin{tabular}{|c|c|c|c|c|c|c|c|c|c|c|}
\hline \multirow[b]{3}{*}{ PCR } & \multicolumn{10}{|c|}{ Gota espessa } \\
\hline & \multicolumn{2}{|r|}{ P.f. } & \multicolumn{2}{|r|}{$P . v$. } & \multicolumn{2}{|c|}{$P f .+P . v$} & \multicolumn{2}{|c|}{ negativa } & \multicolumn{2}{|c|}{ total } \\
\hline & $\mathrm{n}^{0}$ & $\%$ & $\mathrm{n}^{\underline{0}}$ & $\%$ & $\mathrm{n}^{0}$ & $\%$ & $\mathrm{n}^{0}$ & $\%$ & $\mathrm{n}^{0}$ & $\%$ \\
\hline$\overline{P . f .}$ & 59 & 79,7 & 0 & 0,0 & 0 & 0,0 & 65 & 29,4 & 124 & 36,0 \\
\hline P.v. & 0 & 0,0 & 30 & 66,7 & 0 & 0,0 & 30 & 13,6 & 60 & 17,5 \\
\hline$P . f .+P . v$ & 13 & 17,6 & 15 & 33,3 & 4 & 100,0 & 19 & 8,6 & 51 & 14,8 \\
\hline negativa & 2 & 2,7 & 0 & 0,0 & 0 & 0,0 & 107 & 48,4 & 109 & 31,7 \\
\hline Total & 74 & 100,0 & 45 & 100,0 & 4 & 100,0 & 221 & 100,0 & 344 & 100,0 \\
\hline
\end{tabular}

P.f.: Plasmodium falciparum, P.v.: Plasmodium vivax, PCR: reação em cadeia da polimerase.

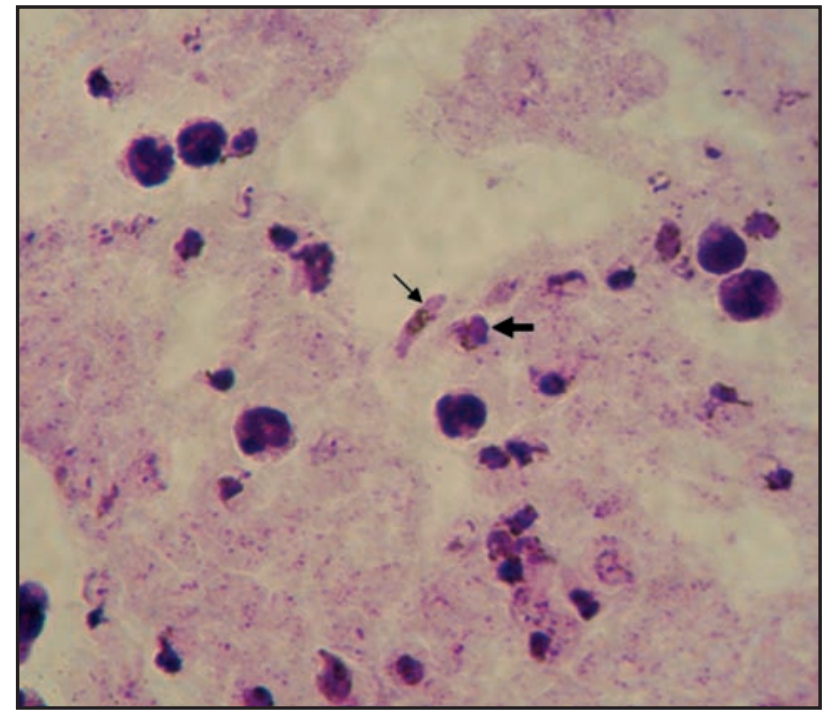

Figura 1 - Lâmina de gota espessa desemoglobinizada com azul de metileno, e corada com Giemsa (1.000x de aumento), utilizada na rotina de diagnóstico de malária. A infecção mista (Plasmodium falciparum/Plasmodium vivax) é evidenciada pela observação de gametócitos de Plasmodium falciparum (seta fina) e formas irregulares de Plasmodium vivax (seta grossa).

\begin{tabular}{|c|c|c|c|c|c|c|c|c|}
\hline & PM & 1 & 2 & 3 & 4 & 5 & 6 & 7 \\
\hline $\begin{array}{l}200 \mathrm{pb}- \\
100 \mathrm{pb}\end{array}$ & $\frac{15}{6}$ & 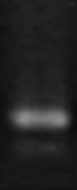 & 20 & & (2) & 0 & & cas \\
\hline
\end{tabular}

Figura $1 B$ - Gel de agarose $2 \%$ corado com brometo de etídeo $(10 \mu \mathrm{g} / \mu \mathrm{L})$, evidenciando infecção mista (P.f./P.v.) após reação de nested-PCR. PM: peso molecular; 1: Infecçao mista $(P f+P v), 2:$ controle positivo de Plasmodium vivax (Sal I); 3: controle negativo; 4: amostra positiva para Plasmodium vivax; 5: controle positivo de Plasmodium falciparum (W2); 6) Diagnóstico molecular negativo, 7) amostra positiva para Plasmodium falciparum, pb: pares de bases. (W2 e Sal I - amostras controle da Indochina e El salvador respectivamente, fonte: MR4).

co-positividade de $96,7 \%$, co-negatividade de $62,2 \%$ e coeficiente kappa de 0,44. Para Plasmodium vivax, co-positividade de 100\%, co-negatividade de 78,1\% e coeficiente kappa de 0,56. Na detecção da malária mista, co-positividade de 100\%, conegatividade de 84,9\% e coeficiente kappa de 0,26.

Nos casos em que a PCR detectou infecção mista, quando o paciente apresentava gota espessa positiva apenas para Plasmodium vivax, a média da parasitemia (à gota espessa) foi semelhante à média dos casos com infecção exclusiva por
Plasmodium vivax, à PCR (3.555 parasitas $/ \mathrm{mm}^{3}$ x 3.881 parasitas $/ \mathrm{mm}^{3}$; teste de Mann-Whitney, $\left.\mathrm{p}=0,23\right)$.

\section{DISCUSSÃO}

A gota espessa é um método sensível capaz de detectar 0,001\% de parasitemia, ou seja, acima de 1 parasita/ $\mu \mathrm{L}$ de sangue ${ }^{31}$. Contudo, o método torna-se pouco sensível quando os parasitas estão presentes em número muito reduzido $(<1 / \mu \mathrm{L}$ de sangue) ou quando o indivíduo está infectado por mais de uma espécie de plasmódio. Inúmeros estudos têm relatado a maior sensibilidade da PCR na detecção de baixas parasitemias ${ }^{3419}$.

Sabe-se que a ocorrência de infecções por mais de uma espécie de plasmódio pode ser ainda maior ${ }^{14}$. Estudos realizados com PCR na Tailândia ${ }^{26}$, Venezuela ${ }^{17}$ e Brasil $^{5}$ demonstraram a ocorrência de infecção mista em proporções acima das evidenciadas à gota espessa.

Estudo realizado na Venezuela demonstrou que 29\% das infecções diagnosticadas à gota espessa como Plasmodium vivax, quando analisadas pela PCR, foram identificadas como infecções mistas por Plasmodium vivax e Plasmodium falciparum ${ }^{17}$. No Irã, estudo recente também demonstrou alta freqüência de infecções mistas não-identificadas à gota espessa ${ }^{7}$. Aproximadamente um terço das amostras positivas para Plasmodium vivax, à gota espessa, na nossa casuística, foram positivas para ambas espécies (Plasmodium falciparum/Plasmodium vivax) à PCR. A validade externa dos resultados encontrados, entretanto, é baixa, pois a freqüência de infecções mistas depende em grande parte das condições epidemiológicas de cada área endêmica e da época em que o estudo foi realizado.

A FMT-AM é uma das unidades de diagnóstico de malária de Manaus, tendo notificado em média 35\% dos casos de malária do município, nos últimos 10 anos. Em 2003, a instituição registrou 30.017 casos de malária, sendo 125 (0,41\%) de infecção mista. Se utilizarmos a PCR como padrão-ouro para o diagnóstico da infecção mista, de acordo com os resultados apresentados neste estudo, estimar-se-ia uma frequiência de infecção mista de 5,3\% neste centro da Amazônia Ocidental Brasileira. Considerando que os resultados apresentados são oriundos de uma unidade de referência para o diagnóstico da malária no Estado do Amazonas, é possível que a quantidade de infecções mistas seja ainda mais sub-notificada na rede descentralizada de diagnóstico.

É necessário, entretanto, questionar se os parasitas detectados pela PCR são da fato implicados no quadro clínico agudo do 
paciente. Em função dos estudos que demonstram ocorrência de até 49,5\% de portadores assintomáticos de plasmódio em Rondônia ${ }^{1}$, existe a possibilidade de que estes portadores crônicos de uma espécie se infectem por outra espécie responsável pelos sintomas clínicos, já que não há proteção clínica cruzada conferida por espécies diferentes. Observou-se também em Rondônia seis vezes mais casos de infecção mista à PCR, em relação à gota espessa, tendo sido esta freqüência menor em outra localidade com menos portadores assintomáticos de plasmódio ${ }^{1}$.

A PCR detectou $10 \%$ de infecções causadas pelo Plasmodium malariae, não identificadas pela microscopia, também em Rondônia, o que foi diferente de nossos resultados 5 . A ocorrência da infecção por esta espécie, em Mato Grosso, chegou a 11,9\%, por PCR $^{22}$. É possível que a distribuição desta espécie não seja homogênea em toda a Amazônia Brasileira.

A evidência de que amostras com infecção mista à PCR não apresentaram maior densidade parasitária em comparação às amostras com monoinfecção reforça a idéia de que nem sempre as gotas espessas positivas para Plasmodium vivax, com alta parasitemia devem ser interpretadas sistematicamente como lâminas de infecção mista, como orientado no passado aos microscopistas de áreas endêmicas.

A padronização de um protocolo único de PCR é um primeiro passo na tentativa de se avaliar o desempenho desta ferramenta da biologia molecular para o diagnóstico da malária, permitindo a comparação dos resultados obtidos em diferentes áreas endêmicas.

A realização da técnica com amostra de sangue total ou com amostra de sangue total em papel de filtro parece não interferir de maneira importante nos resultados, sendo que os trabalhos de campo, em geral, se beneficiam da coleta da amostra em papel de filtro, pela simplicidade de armazenamento e transporte até 0 laboratório onde o exame será realizado ${ }^{23}$.

Mais recentemente, a técnica de PCR em tempo real mostrou poder contribuir não apenas para diminuir as chances de contaminação das amostras, mas também para diminuir os custos e o tempo para a obtenção dos resultados'.

A alta co-positividade da PCR com a gota espessa na detecção de infecção malárica reforça sua utilidade em estudos epidemiológicos de prevalência, estudos de eficácia terapêutica e vacinal, detecção de portadores assintomáticos de plasmódio e na avaliação de novos métodos diagnósticos para malária ${ }^{101521}$. Em bancos de sangue, o emprego da PCR pode ser de suma importância para o controle da malária transfusional ${ }^{8}$. Finalmente, o uso da PCR em unidades de referência pode ter alguma utilidade na avaliação do desempenho de microscopistas considerados experientes ${ }^{6}$.

A busca de novas alternativas diagnósticas para a malária é uma das metas do Programa Nacional de Controle da Malária (PNCM) do Ministério da Saúde, mas é preciso cautela ao escolher a PCR como teste padrão-ouro na comparação com outros testes diagnósticos da doença clínica, já que amplificando DNA, a PCR pode diagnosticar, p. ex., a presença apenas de formas sexuadas (gametócitos), não se prestando, portanto, para o diagnóstico exclusivo das formas consideradas patogênicas.
Os resultados apresentados sugerem que as infecções mistas na Amazônia Brasileira podem estar subestimadas e que a PCR pode ser uma importante ferramenta de diagnóstico da infecção pelo plasmódio, especialmente em condições nas quais a gota espessa apresenta pior desempenho. Não foram identificadas infecções por Plasmodium malariae nesta amostra estudada, em Manaus.

\section{AGRADECIMENTOS}

Os autores agradecem ao Programa de Pós-Graduação em Doenças Tropicais e Infecciosas da Universidade do Estado do Amazonas, aos técnicos da Gerência de Malária da Fundação de Medicina Tropical do Amazonas, especialmente ao Sr. José Eckner Lessa, Sr. Jubal Gonzaga Simões, pela revisão das gotas espessas, e à Sra. Raimunda Ericilda Soares de Araújo, pela coleta de amostras e extração de DNA.

\section{REFERÊNCIAS}

1. Alves FP, Durlacher RR, Menezes MJ, Krieger H, Silva LH, Camargo EP. High prevalence of asymptomatic Plasmodium vivax and Plasmodium falciparum infections in native Amazonian populations. The American Journal of Tropical Medicine and Hygiene 66:641-648, 2002.

2. Barker Jr RH, Banchongaksorn T, Courval JM, Suwonkerd W, Rimwungtragoon K, Wirth DF. A simple method to detect Plasmodium falciparum directly from blood samples using the polymerase chain reaction. The American Journal of Tropical Medicine and Hygiene 46:416-426, 1992.

3. Black J, Hommel M, Snounou G, Pinder M. Mixed infections with Plasmodium falciparum and P. malariae and fever in malaria. Lancet 343:1095, 1994.

4. Brown AE, Kain KC, Pipithkul J, Webster HK. Demonstration by the polymerase chain reaction of mixed Plasmodium falciparum and $P$. vivax infections undetected by conventional microscopy. Transactions of the Royal Society of Tropical Medicine and Hygiene 86:609-612, 1992.

5. Cavasini MT, Ribeiro WL, Kawamoto F, Ferreira MU. How prevalent is Plasmodium malariae in Rondonia, western Brazilian Amazon? Revista da Sociedade Brasileira de Medicina Tropical 33:489-492, 2000.

6. Di Santi SM, Kirchgatter K, Brunialti KC, Oliveira AM, Ferreira SR, Boulos M. PCR - based diagnosis to evaluate the performance of malaria reference centers. Revista do Instituto de Medicina Tropical de São Paulo 46:183-187, 2004.

7. Ebrahimzadeh A, Fouladi B, Fazaeli A. High rate of detection of mixed infections of Plasmodium vivax and Plasmodium falciparum in South-East of Iran, using nested PCR. Parasitology International 56:61-64, 2007.

8. Fugikaha E, Fornazari PA, Penhalbel RS, Lorenzetti A, Maroso RD, Amoras JT, Saraiva AS, Silva RU, Bonini-Domingos CR, Mattos LC, Rossit AR, Cavasini CE, Machado RL. Molecular screening of Plasmodium sp. asymptomatic carriers among transfusion centers from Brazilian Amazon region. Revista do Instituto de Medicina Tropical de São Paulo 49:1-4, 2007.

9. Gama BE, Silva-Pires FD, Lopes MN, Cardoso MA, Britto C, Torres KL, Mendonca Lima L, Souza JM, Daniel-Ribeiro CT, Fatima Ferreira-da-Cruz M. Real-time PCR versus conventional PCR for malaria parasite detection in low-grade parasitemia. Experimental Parasitology 116: 427-432, 2007.

10. Imoukhuede EB, Andrews L, Milligan P, Berthoud T, Bojang K, Nwakanma D, Ismaili J, Buckee C, Njie F, Keita S, Sowe M, Lang T, Gilbert SC, Greenwood BM, Hill AV. Low-level malaria infections detected by a sensitive polymerase chain reaction assay and use of this technique in the evaluation of malaria vaccines in an endemic area. The American Journal of Tropical Medicine and Hygiene 76:486-493, 2007.

11. Kimura M, Kaneko O, Inoue A, Ishii A, Tanabe K. Amplification by polymerase chain reaction of Plasmodium falciparum DNA from Giemsa-stained thin blood smears. Molecular and Biochemical Parasitology 70:193-197, 1995. 
12. Laserson KF, Petralanda I, Hamlin DM, Almera R, Fuentes M, Carrasquel A, Barker Jr RH. Use of the polymerase chain reaction to directly detect malaria parasites in blood samples from the Venezuelan Amazon. The American Journal of Tropical Medicine and Hygiene 50:169-180, 1994.

13. Lorenzetti A, Fornazari PA, Bonini-Domingos AC, Souza Rodrigues Penhalbel R, Fugikaha E, Bonini-Domingos CR, Fraga VD, Conceição LM, Rossit AR, Cavasini CE, Couto VS, Machado RL. Mixed Plasmodium falciparum infections and its clinical implications in four areas of the Brazilian Amazon region. Acta Tropica 107:8-12, 2008.

14. Mayxay M, Pukrittayakamee S, Newton PN, White NJ. Mixed-species malaria infections in humans. Trends in Parasitology 20:233-240, 2004.

15. Mens P, Spieker N, Omar S, Heijnen M, Schallig H, Kager PA. Is molecular biology the best alternative for diagnosis of malaria to microscopy? A comparison between microscopy, antigen detection and molecular tests in rural Kenya and urban Tanzania. Tropical Medicine and International Health 12:238-244, 2007.

16. Ministério da Saúde. Manual de diagnóstico laboratorial da malária. Acessado em 26 de dezembro de 2006. Disponível em http://portal.saude.gov.br/portal/ arquivos/pdf/manual_diag_malaria.pdf, 2005

17. Morassin B, Fabre R, Berry A, Magnaval JF. One year's experience with the polymerase chain reaction as a routine method for the diagnosis of imported malaria. American Journal of Tropical Medicine and Hygiene 66:503-508, 2002.

18. Postigo M, Mendoza-Leon A, Perez HA. Malaria diagnosis by the polymerase chain reaction: a field study in south-eastern Venezuela. Transactions of the Royal Society of Tropical Medicine and Hygiene 92:509-511, 1998.

19. Purnomo AS, Gomez-Saladin E, Bangs MJ. Rare quadruple malaria infection in Irian Jaya Indonesia. Journal of Parasitology 85:574-579, 1999.

20. Rakotonirina H, Barnadas C, Raherijafy R, Andrianantenaina H, Ratsimbasoa A, Randrianasolo L, Jahevitra M, Andriantsoanirina V, Menard D. Accuracy and reliability of malaria diagnostic techniques for guiding febrile outpatient treatment in malaria-endemic countries. The American Journal of Tropical Medicine and Hygiene 78:217-221, 2008.

21. Rodulfo H, Donato M, Mora R, Gonzalez L, Contreras CE. Comparison of the diagnosis of malaria by microscopy, immunochromatography and PCR in endemic areas of Venezuela. Brazilian Journal of Medical and Biological Research 40:535-543, 2007.

22. Scopel KK, Fontes CJ, Nunes AC, Horta MF, Braga EM. High prevalence of Plamodium malariae infections in a Brazilian Amazon endemic area (Apiacas-
Mato Grosso State) as detected by polymerase chain reaction. Acta Tropica 90:61-64, 2004.

23. Singh B, Bobogare A, Cox-Singh J, Snounou G, Abdullah MS, Rahman HA. A genus- and species-specific nested polymerase chain reaction malaria detection assay for epidemiologic studies. The American Journal of Tropical Medicine and Hygiene 60:687-692, 1999.

24. Singh B, Cox-Singh J, Miller AO, Abdullah MS, Snounou G, Rahman HA. Detection of malaria in Malaysia by nested polymerase chain reaction amplification of dried blood spots on filter papers. Transactions of the Royal Society of Tropical Medicine and Hygiene 90:519-521, 1996.

25. Snounou G, Viriyakosol S, Zhu XP, Jarra W, Pinheiro L, Rosario VE, Thaithong S, Brown KN. High sensitivity of detection of human malaria parasites by the use of nested polymerase chain reaction. Molecular and Biochemical Parasitology 61:315-320, 1993.

26. Tirasophon W, Ponglikitmongkol M, Wilairat P, Boonsaeng V, Panyim S. A novel detection of a single Plasmodium falciparum in infected blood. Biochemical and Biophysical Research Communications 175:179-184, 1991.

27. Torres KL, Figueiredo DV, Zalis MG, Daniel-Ribeiro CT, Alecrim W, Ferreira-daCruz MF. Standardization of a very specific and sensitive single PCR for detection of Plasmodium vivax in low parasitized individuals and its usefulness for screening blood donors. Parasitology Research 98:519-524, 2006.

28. Vieira PP, Ferreira MU, Alecrim MG, Alecrim WD, da Silva LH, Sihuincha MM, Joy DA, Mu J, Su XZ, Zalis MG. pfcrt Polymorphism and the spread of chloroquine resistance in Plasmodium falciparum populations across the Amazon Basin. Journal Infectious Diseases 190: 417-424, 2004.

29. Vinetz JM, Li J, McCutchan TF, Kaslow DC. Plasmodium malariae infection in an asymptomatic 74-year-old Greek woman with splenomegaly. The New England Journal of Medicine 338:367-371, 1998.

30. Vu TT, Tran VB, Phan NT, Le TT, Luong VH, O'Brien E, Morris GE. Screening donor blood for malaria by polymerase chain reaction. Transactions of the Royal Society of Tropical Medicine and Hygiene 89:44-47, 1995.

31. Wataya Y, Arai M, Kubochi F, Mizukoshi C, Kakutani T, Ohta N, Ishii A. DNA diagnosis of falciparum malaria using a double PCR technique: a field trial in the Solomon Islands. Molecular and Biochemical Parasitology 58:165-167, 1993.

32. Zalis MG, Ferreira-da-Cruz MF, Balthazar-Guedes HC, Banic DM, Alecrim W, Souza JM, Druilhe P, Daniel-Ribeiro CT. Malaria diagnosis: standardization of a polymerase chain reaction for the detection of Plasmodium falciparum parasites in individuals with low-grade parasitemia. Parasitology Research 82: 612-616, 1996. 